\title{
Avances en la terapia inhalatoria de las vías aéreas en asma y EPOC
}

\author{
EDGARDO CARRASCO C.*
}

\section{Advances in the inhalation therapy of the airways in asthma and COPD}

The inhalers are the most efficient and safest form of treatment in stable bronchial obstructive disease: Asthma and COPD. There are two types of inhalers: a) dose metered inhalers (MDI) for the administration of bronchodilator drugs $\left(\beta_{2}\right.$ agonists and anti-cholinergics) and corticosteroids dissolved in a HFA propellent, and b) dry powder inhalers (DPI) that are activated by the inspiratory flow of the patient, without need of an aero-chamber. The clinical prescription of inhaled bronchodilators and corticosteroids will depend on their pharmacological activity, dosing, long of action and the possibility of association with other drugs that empower the therapeutic result. The short acting $\beta_{2}$ agonists (SABA) last 4 hours, while the long acting ones (LABA) extend their effect to 12-24 hours. In persistent asthma the combination of an inhaled corticosteroid with a long acting $\beta_{2}$ agonist will control $95 \%$ of patients. A new combination of mometasone with formoterol will help in this endeavour. Anti-cholinergic medication dilate the airways by a different mechanism, and are short (SAMA) and long lasting (LAMA) Ipratropium is a short action anti cholinergic drug and therapeutically is limited to mild COPD (Gold A). The long action anti cholinergic, as tiotropium and glicopirronium, works for 24 hours and are useful in moderate to severe COPD (Gold $B$ and $C$ ), either alone or associated to a 24 hours acting $\beta_{2}$ agonist (LABA). Another option in this category of COPD is the combination of an inhaled corticosteroid inhaler (CEI) with a LABA, taking into account that could increase the occurrence of pneumonia or reactivation of tuberculosis. In the more severe COPD patients (Gold D) the actual tendency is the use of a triple association: LAMA (tiotropium or glicopirronium) + a 24 hours LABA + CEI. The association of glicopirronium with indacaterol has been shown to be as useful as the triple associated therapy.

Key words: Inhalation therapy, asthma, COPD, classification, review new treatments.

\section{Resumen}

Los inhaladores son la forma más eficiente y segura de administración de la terapia en las enfermedades bronquiales obstructivas: Asma y EPOC, en fase estable. Los inhaladores son de dos tipos: a) inhaladores de dosis medidas (IDM), en que el fármaco es administrado con un propelente de tipo HFA, y b) inhaladores de polvo seco, que son activados por la inspiración del paciente y no requieren de aero-cámara. Las aplicaciones clínicas de los broncodilatadores y corticoides inhalados (CEI), dependerán del tipo de fármaco y sus dosis unitarias, de su mecanismo de acción, y las posibilidades de emplear asociaciones que potencien su acción terapéutica. Los agonistas $\beta_{2}$ adrenérgicos de acción corta (SABA) limitan su acción a $4 \mathrm{~h}$, y los de larga acción (LABA) pueden actuar por 12 a $24 \mathrm{~h}$. En el asma persistente (leve, moderado, severo) la combinación de un corticoide inhalado con un LABA controlará el 95\% de los pacientes. Una nueva combinación de mometasona con formoterol ayudará a estos efectos. Los anticolinérgicos producen broncodilatación por un mecanismo diferente a los $\beta$ agonistas, y son de acción corta (SAMA) y prolongada (LAMA). El ipratropio es un anticolinérgico de acción corta (4 h) que tiene un empleo limitado en la EPOC leve. Los anticolinérgicos de acción prolongada (tiotropio y glicopirronio) tienen actividad por 24 hy son útiles en las EPOC moderadas y severas (Gold B y C), ya sea solos o combinados con un beta aagonista ultra prolongado (indacaterol).

* Profesor titular. Facultad de Medicina, Universidad de Chile. 
Otra opción es un CEI + un LABA, pero aumenta frecuencia de neumonías y reactivaciones de tuberculosis. En los pacientes EPOC más severos (Gold D) la tendencia actual es emplear un tratamiento triasociado: LAMA (tiotropio o glicopirronio) + LABA de $24 h+$ C.E.I. La asociación de glicopirronio + Indacaterol ha demostrado ser tan eficaz como el tratamiento triasociado.

Palabras clave: Terapia inhalatoria, asma, EPOC, clasificación, revisión de nuevos tratamientos.

\section{Características de los inhaladores}

\section{Inhaladores presurizados de dosis medidas (IDM)}

Son los más usados en nuestro medio, y en el contenedor del dispositivo se encuentran él o los fármacos (beta adrenérgicos, corticoesteroides, anticolinérgicos) junto a un propelente (HFA) y lubricantes. El medicamento se entrega cuando se activa el dispositivo, abriendo una válvula que libera una dosis medida y conocida del fármaco en aerosol. La eficiencia del inhalador presurizado depende del tamaño de las partículas que componen el aerosol, lo que determina el depósito de la droga en las distintas zonas de la vía aérea. Con el cambio del propelente de clorofluorocarbono (CFC) a hidrofluoroalcano (HFA), se ha logrado que se deposite más profundamente en la vía aérea con una concentración superior al $50 \%$.

El mayor depósito de la droga en el pulmón se logra: a) ubicando el inhalador a $4 \mathrm{~cm}$ de la boca (si es que no se usa un espaciador); b) actuando el inhalador al comienzo de una inhalación; c) inspirando con un flujo moderado, igual a $1 \mathrm{~L} / \mathrm{s}$ para disminuir la inercia de las partículas, d) efectuando una pausa respiratoria de 5 a 10 segundos para lograr una mejor distribución del aerosol. El uso de flujos inspiratorios muy altos y la actuación del inhalador dentro de la boca aumenta el depósito del aerosol en la faringe, su deglución y un incremento de la cantidad de fármaco absorbido hacia la circulación.

\section{Inhaladores de polvo seco}

Los inhaladores de polvo seco permiten entregar los mismos fármacos con algunas ventajas derivadas de esta forma de administración. Inicialmente se emplearon inhaladores que usaban cápsulas de gelatina que se colocaban manualmente en el dispositivo, que tenía un gatillo para perforar la cápsula, aspirándose el polvo con un torbellino (Spinhaler). Para superar estos problemas se desarrollaron envases que contienen un blíster con multidosis, que permite cargar el medicamento para su uso con una simple rotación, que deja una dosis de droga disponible para su inhalación sin requerir un espaciador. El manejo de estos inhaladores requiere flujos inspiratorios variables, de acuerdo con la diferente resistencia interna que presentan.

Tienen la ventaja de menos efectos secundarios que los IDM que proporcionan $\beta$-2 agonistas adrenérgicos y menos disfonía que los que usan corticoesteroides inhalados. En Chile tenemos disponible el turbohaler ${ }^{\circledR}$ que combina formoterol con budesonida (Symbicort $\left.{ }^{\circledR}\right)$; Diskhaler ${ }^{\circledR}$ que combina salmeterol con fluticasona (Seretide $\left.{ }^{\circledR}\right)$, el Handihaler ${ }^{\circledR}$ y Respimat ${ }^{\circledR}$ que contienen tiotropio (Spiriva $\left.{ }^{\circledR}\right)$, el twisthaler que contiene mometasona (Momexa $\left.{ }^{\circledR}\right)$ y el breezhaler que libera indacaterol (Onbrize ${ }^{\circledR}$ ) o glicopirronio (Seebri $\left.{ }^{\circledR}\right)$, ya sea solos o en combinación (Tablas 1, 2 y 3 ).

\section{Aplicaciones clínicas de los fármacos inhalados}

\section{Broncodilatadores}

Los agonistas $\beta_{2}$ adrenérgicos y los anticolinérgicos son broncodilatadores y aumentan el calibre de las vías aéreas al relajar el músculo liso bronquial.

\section{A. Agonistas $\beta_{2}$ adrenérgicos}

Llamados también simpaticomiméticos, porque actúan similarmente a los estimuladores de las fibras adrenérgicos post-ganglionares, se clasifican de acuerdo con su duración de acción en $\beta_{2}$ adrenérgicos de acción corta y $\beta_{2}$ adrenérgicos de acción prolongada.

\section{a) $\beta_{2}$ adrenérgicos de acción corta (SABA)}

Están constituidos por el salbutamol, la terbutalina y el fenoterol.

Estos fármacos son hidrófilos y permanecen exclusivamente en la fase acuosa que rodea la membrana epitelial lo que permite su difusión rápida al receptor beta adrenérgico, pero permanecen poco tiempo en la membrana. Esto explica su inicio rápido y el poco tiempo que dura su acción broncodilatadora.

Administrados por inhalación, su acción comienza a los 2-3 min, producen una marcada broncodilatación a los $15 \mathrm{~min}$, con un efecto máximo a los 60-90 min, persistiendo por 4 a $6 \mathrm{~h}$. 
Existen presentaciones para inhalador presurizado, polvo seco y soluciones para nebulizaciones.

En los pacientes con EPOC con síntomas leves se recomienda el uso de broncodilatadores de acción corta para el alivio de los síntomas y aumentar tolerancia al esfuerzo. En los asmáticos, cualquiera que sea su severidad, se recomienda el $\beta_{2}$ agonista de acción corta para el alivio rápido de los síntomas (recomendación A). Los $\beta_{2}$ agonistas de acción corta administrados 15 min antes del ejercicio son el medicamento de elección para prevenir la broncoconstricción inducida por el ejercicio (Tabla $1 \mathrm{~A}$ con dosis de $\beta_{2}$ agonistas de acción corta).

Tabla 1. Dosis diarias de broncodilatadores $\beta$-2 agonistas en aerosol en adultos

A. Broncodilatadores de acción rápida y corta

\begin{tabular}{|lccc|}
\hline Dosis única y presentación & $\begin{array}{c}\text { Dosis diaria } \\
\text { asma estable }\end{array}$ & $\begin{array}{c}\text { Dosis en exacerbación } \\
\text { de asma }\end{array}$ & $\begin{array}{c}\text { Dosis en asma } \\
\text { por ejercicio }\end{array}$ \\
$\begin{array}{l}\text { Salbutamol } \\
(100 \mu \mathrm{g} x \text { inhalación) }\end{array}$ & $\begin{array}{c}200 \mu \mathrm{g} \mathrm{PRN} \text { ó } \\
200 \mu \mathrm{g} \mathrm{c} / 8 \mathrm{~h}\end{array}$ & $\begin{array}{c}200 \mu \mathrm{g} \mathrm{c} / 15 \mathrm{~min} \\
\text { por hasta } 2 \mathrm{~h}\end{array}$ & $\begin{array}{c}200 \mu \mathrm{g} 15 \mathrm{~min} \text { antes } \\
\text { ejercicio }\end{array}$ \\
$\begin{array}{l}\text { Salbutamol }+ \text { bromuro de ipratropio } \\
(100 \mu \mathrm{g}+20 \mu \mathrm{g} \text { x inhalación) }\end{array}$ & $\begin{array}{c}200+40 \mu \mathrm{g} \text { PRN } \\
\mathrm{o} / 6-8 \mathrm{~h})\end{array}$ & $\begin{array}{c}200+40 \mu \mathrm{g} \mathrm{c} / 15 \mathrm{~min} \\
\text { por } 2 \mathrm{~h}\end{array}$ & $\begin{array}{c}200+40 \mu \mathrm{g} 15 \mathrm{~min} \\
\text { antes de ejercicio }\end{array}$ \\
$\begin{array}{l}\text { Fenoterol }+ \text { bromuro de Ipratropio } \\
(50 / 20 \mu \mathrm{g} \text { x inhalación) }\end{array}$ & $\begin{array}{c}100+40 \mu \mathrm{g} \text { PR o } \\
\mathrm{c} / 6-8 \mathrm{~h}\end{array}$ & $\begin{array}{c}100+40 \mu \mathrm{g} \mathrm{c} / 15 \mathrm{~min} \\
\text { por } 2 \mathrm{~h}\end{array}$ & $\begin{array}{c}100+40 \mu \mathrm{g} 15 \mathrm{~min} \\
\text { antes del ejercicio }\end{array}$ \\
\hline
\end{tabular}

\section{B. Broncodilatadores de acción prolongada}

\begin{tabular}{|c|c|c|c|}
\hline Dosis única y presentación & Dosis diarias & Dosis en exacerbación & $\begin{array}{c}\text { Dosis en asma } \\
\text { por ejercicio }\end{array}$ \\
\hline $\begin{array}{c}\text { Salmeterol* } \\
25 \mu \mathrm{g} \times \text { inhalación }\end{array}$ & $\begin{array}{c}50 \mu \mathrm{g} \text { dividido en } 2 \text { dosis } \\
\text { asociado a CEI }\end{array}$ & $\begin{array}{c}50 \mu \mathrm{g} \text { dividido en } 2 \text { dosis } \\
\text { y asociado a CEI }\end{array}$ & $\begin{array}{c}50 \mu \mathrm{g} 15 \text { min antes de } \\
\text { ejercicio }\end{array}$ \\
\hline $\begin{array}{c}\text { Formoterol } \\
4,5 \mu \mathrm{g} \times \text { inhalación } \\
9,0 \mu \mathrm{g} \times \text { inhalación* }\end{array}$ & $\begin{array}{l}9 \mu \mathrm{g} \text { dividido en } 2 \text { dosis } \\
+ \text { CEI } \\
18 \mu \mathrm{g} \text { dividido en } 2 \text { dosis } \\
\text { en EPOC }\end{array}$ & $\begin{array}{c}9 \mu \mathrm{g}+\text { Budesonida } \\
3-4 \text { v/día (rescate) } \\
\text { Ibid } \\
\text { Uso en EPOC }\end{array}$ & $\begin{array}{c}9 \mu \mathrm{g} 15 \min \text { antes de } \\
\text { ejercicio }\end{array}$ \\
\hline $\begin{array}{l}\text { Indacaterol } \\
150-300 \mu \mathrm{g}^{*}\end{array}$ & $\begin{array}{c}150 \text { ó } 300 \mu \mathrm{g} \mathrm{c} / 24 \mathrm{~h} \text { en } \\
\text { EPOC }\end{array}$ & No aplicable & No aplicable \\
\hline
\end{tabular}

*Uso aislado aplicable sólo en EPOC. No pueden usarse en asma como monoterapia, por peligro de reacciones adversas severas. Uso permitido si se asocia a un corticoesteroide inhalado, ojalá en un solo dispositivo contenedor. CEI: corticoesteroide inhalado.

Tabla 2. Anticolinérgicos inhalados en obstrucción de vías aéreas

\begin{tabular}{|c|c|c|c|}
\hline $\begin{array}{l}\text { Dosis única y } \\
\text { presentación }\end{array}$ & $\begin{array}{c}\text { Dosis diaria } \\
\text { (dividida en dosis) }\end{array}$ & Dosis en EPOC estable & Otras aplicaciones \\
\hline $\begin{array}{l}\text { Bromuro de Ipratropio } \\
20 \mu \mathrm{g} \text { x inhalación (IDM) } \\
\text { Acción corta }\end{array}$ & $40 \mathrm{a} \mu \mathrm{g} \mathrm{c} / 8 \mathrm{~h}$ & $\begin{array}{l}60 \mu \mathrm{g} \text { asociado a fenoterol } \\
(100 \mu \mathrm{g}) \mathrm{c} / 15 \mathrm{~min}\end{array}$ & No aplicable \\
\hline $\begin{array}{l}0,25 \mu \mathrm{g} / \mathrm{ml} \\
\text { Para NBZ }\end{array}$ & & & No aplicable \\
\hline $\begin{array}{l}\text { Tiotropio } \\
\text { cápsulas } 18 \mu \mathrm{g} \\
\text { (handihaler) IPS }\end{array}$ & $\begin{array}{l}1 \text { cápsula inhalada } \\
\mathrm{c} / 24 \mathrm{~h}\end{array}$ & $\begin{array}{l}1 \text { cápsula c/ } 24 \text { h sola o asociada } \\
\text { a LABA en EPOC leve a } \\
\text { moderado }\end{array}$ & $\begin{array}{l}\text { Uso en asma severo } \\
\text { asociado a CEI y LABA }\end{array}$ \\
\hline $\begin{array}{l}\text { Tiotropio respimat } \\
\text { solución } 2,5 \mu \mathrm{g} \text { por } \\
\text { inhalación IPS }\end{array}$ & $\begin{array}{c}5 \mu \mathrm{g} \\
(2 \text { inhalaciones c/24 h) }\end{array}$ & $\begin{array}{l}\text { Dos inhalaciones } 1 \text { vez al día } \\
\text { en EPOC leve o asociado a } \\
\text { LABA en EPOC moderada }\end{array}$ & $\begin{array}{l}\text { Asociado a LABA y CEI en } \\
\text { EPOC severo }\end{array}$ \\
\hline $\begin{array}{l}\text { Glicopirronio (bromuro) } \\
50 \mu \mathrm{g} \text { por inh (IPS) }\end{array}$ & $\begin{array}{l}50 \mu \mathrm{g} 1 \text { vez al día } \\
\text { con breezhaler }\end{array}$ & $\begin{array}{l}\text { En EPOC moderado: una inha- } \\
\text { lación asociada a indacaterol }\end{array}$ & $\begin{array}{l}\text { En EPOC severo uso } \\
\text { asociado a indacaterol y CEI }\end{array}$ \\
\hline
\end{tabular}

IDM: inhalador presurizado dosis medida; NBZ: nebulización; IP: Inhalador polvo seco; CEI; corticoide inhalado. 
b) $\beta_{2}$ adrenérgicos de acción prolongada (LABA)

Representados por salmeterol, formoterol, indacaterol, olodaterol y vilanterol tienen una gran selectividad por los receptores $\beta$ adrenérgicos y mantienen la broncodilatación por $12 \mathrm{~h}$ los dos primeros y por $24 \mathrm{~h}$ los tres últimos. Este tipo de broncodilatador tiene una estructura molecular diferente a los de acción corta. El salmeterol (Serevent $\left.{ }^{\circledR}\right)$, derivado del salbutamol, tiene una gran acción lipofílica. Esto permite que la mayor parte del fármaco pase más lentamente la bicapa lipídica de la membrana celular, lo que explica su inicio de acción más lento (20 min) en relación al formoterol e indacaterol (3 a $5 \mathrm{~min}$ ). El formoterol es moderadamente lipofílico, por lo que penetra la capa lipídica más rápido (menos retención que el salmeterol). Posteriormente ambos permanecen más tiempo en la fase acuosa estimulando por largo tiempo al receptor (12 a $14 \mathrm{~h})$. El indacaterol mantiene su efecto por 24 $\mathrm{h}$, y ello permite su uso 1 vez al día. El uso aislado de broncodilatadores se ha prohibido en el tratamiento del asma persistente al comprobarse en varios estudios clínicos una acción broncoespástica severa por producción de tolerancia de los $\beta$ receptores, que no responden al deseado efecto broncodilatador. Su uso por lo tanto sólo se acepta en combinación con los corticoides inhalados: fluticasona (propionato y furoato), budesonida y mometasona (furoato). A pesar de la restricción de su uso aislado en asma, el salmeterol se ha empleado como monoterapia en la EPOC leve a moderada dado que mejora significativamente el $\mathrm{VEF}_{1}$, disnea y calidad de vida y disminuye las exacerbaciones (evidencia A).

El indacaterol es un $\beta_{2}$ adrenérgico de acción prolongada (hasta $24 \mathrm{~h}$ ) que se presenta como polvo seco liberado desde el dispositivo breezhaler, y para uso en la EPOC solamente. Se proporciona en cápsulas de polvo seco que suministran 150 ó $300 \mu \mathrm{g}$ por inhalación, siendo esta última la dosis diaria máxima. Su efecto broncodilatador es significativamente mayor que el del formoterol y salmeterol y similar al tiotropio (evidencia A), por lo cual es más efectivo para disminuir los síntomas de la EPOC. Además tiene una actividad significativa sobre la disnea, estado de salud y exacerbaciones. Como hecho llamativo puede producir tos transitoria en $24 \%$ de los pacientes ${ }^{1}$.

Como ocurre con otros tratamientos inhalatorios con $\beta_{2}$ agonistas, la administración de indacaterol puede causar ocasionalmente un

Tabla 3. Dosis diarias equivalentes para los glucocorticoides en aerosol y sus combinaciones en adultos

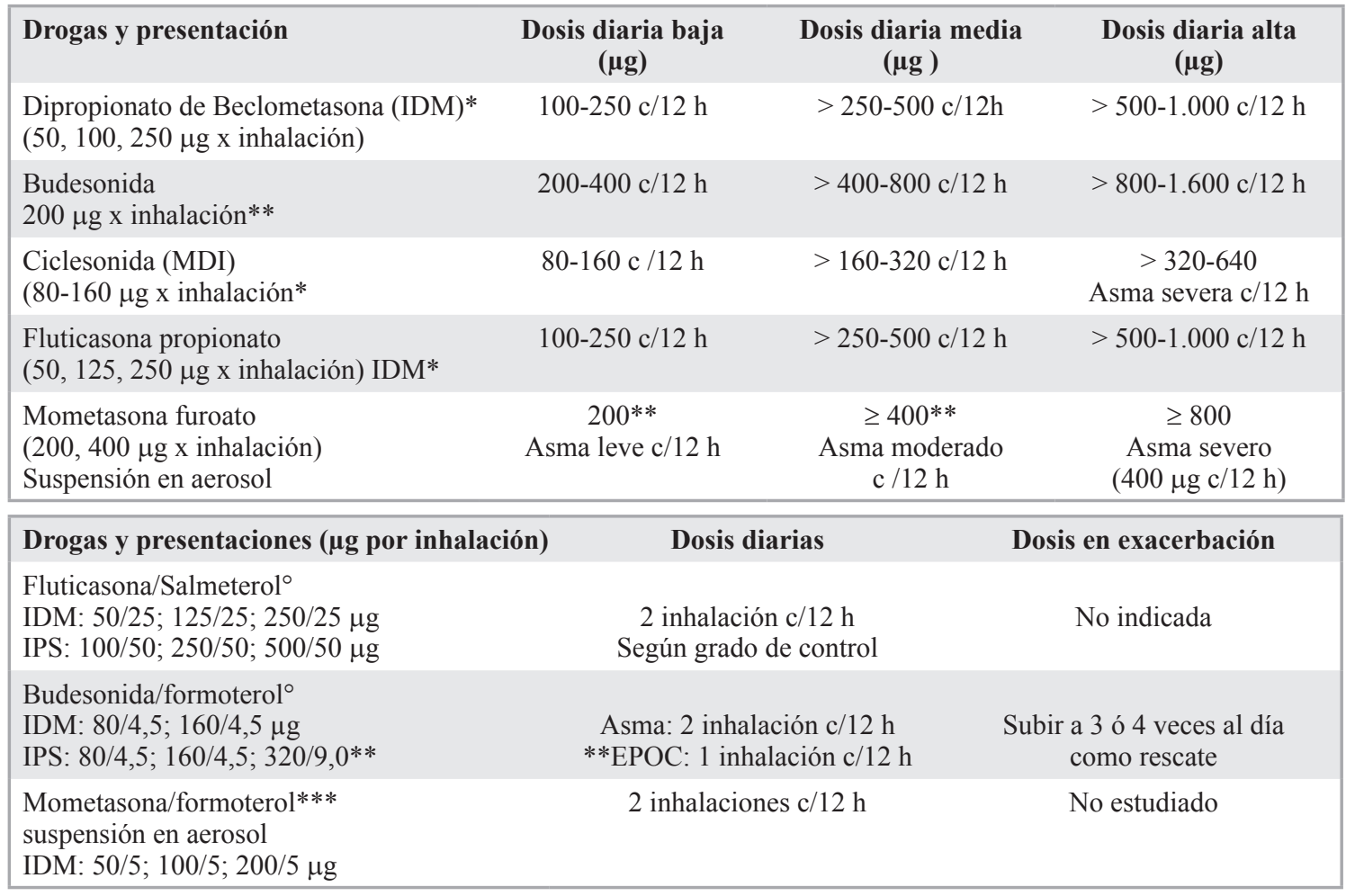

*IDM: inhalador presurizado dosis medida; **IPS: Inhalador polvo seco; ${ }^{\circ} \mathrm{u}$ otro genérico aprobado; ***mayores de 12 años. 
broncoespasmo paradójico. El medicamento debe administrarse con precaución en pacientes con trastornos cardiovasculares, particularmente en insuficiencia coronaria, arritmias e hipertensión arterial; cuadros convulsivos y tirotoxicosis. Igualmente debe usarse con cautela, su asociación a medicamentos que combinen otros $\beta_{2}$ agonistas prolongados a corticoides inhalados, $\mathrm{y}$ medicamentos que prolonguen el espacio QT (Tabla1 B). Aunque no están aún disponibles en nuestro país, el arsenal de beta agonistas de acción prolongada se ha enriquecido con la aprobación por la FDA en enero de 2013 del Olodaterol y en mayo de 2013 del Vilanterol, para uso aislado y en combinación con tiotropio y furoato de fluticasona respectivamente, pero solamente para pacientes con EPOC.

\section{B. Anticolinérgicos}

Los anticolinérgicos son un grupo de fármacos utilizados en el tratamiento de la obstrucción bronquial porque reducen la hipersecreción de moco, inhiben la bronco-constricción refleja colinérgica y atenúan el tono vagal de la vía aérea.

El sistema parasimpático es el principal regulador del tono bronquial, y su aumento de actividad produce constricción del músculo liso bronquial y libera moco dentro de las vías aéreas. Estas acciones están mediadas por la acción de la acetilcolina sobre los receptores muscarínicos, de los cuáles hay tres tipos en el árbol bronquial: M 1, M 2 y M 3. Los anticolinérgicos bloquean la acción de la acetilcolina sobre receptores 1 y 3. La atropina fue el primero de estos agentes utilizados, pero sus efectos indeseables, fundamentalmente cardiovasculares, la dejó de lado. La modificación de su molécula permitió el desarrollo de los anticolinérgicos derivados del amonio cuaternario: el bromuro de oxitropio, el bromuro de ipratropio, y el tiotropio que tienen propiedades farmacológicas similares a la atropina, pero al ser inhalados presentan una absorción sistémica muy pequeña y un buen efecto sobre el tracto respiratorio, sin producir efectos secundarios de significación.

\section{B1) Anticolinérgicos de acción corta (SAMA)}

El bromuro de Ipratropio, disponible en el país desde hace varios años, al ser utilizado en inhalación, inicia su acción broncodilatadora a los 5 min y alcanza su efecto máximo a los 30-60 min, manteniendo su eficacia por 3 a 6 h. Está disponible bajo la forma de inhalador presurizado y solución para nebulizaciones, sólo o combinado con fenoterol. Su acción se debe a la inhibición de los 3 subtipos de receptores muscarínicos. Su mayor empleo se encuentra en la EPOC, donde reduce síntomas y mejora levemente la función pulmonar. En las exacerbaciones de asma se le ha utilizado cuando hay poca respuesta a $\operatorname{los} \beta_{2}$ agonistas de acción corta. Estos medicamentos se encuentran disponibles en GES (Tabla 2).

\section{B2) Anticolinérgicos de acción prolongada (LAMA)}

Estos anti-colinérgicos poseen una potencia muy superior al ipratropio y una selectividad cinética única, que les permiten mantener broncodilatación por $24 \mathrm{~h}$.

Tiotropio (Spiriva $\left.{ }^{\circledR}\right)$. Se usa desde hace 10 años, se administra cada $24 \mathrm{~h}$ en cápsulas que contienen $18 \mu \mathrm{g}$ de polvo seco del fármaco, que se inhalan desde un handihaler, que perfora la cápsula antes de su inhalación bucal sin requerir un espaciador ${ }^{2,3}$. También está disponible una formulación nebulizada (Respimat), bajo la forma de un dispositivo que contiene una solución del fármaco, y que aporta $2,5 \mu \mathrm{g}$ por acción, siendo la dosis recomendada 2 inhalaciones $(5 \mu \mathrm{g})$ una vez al día. Es una opción al polvo seco, inicialmente con restricción de su empleo por eventuales mayores efectos adversos cardiovasculares que la formulación de polvo seco (Handihaler) ${ }^{4}$, situación que se reevaluó recientemente en el estudio Tiospir, que no mostró diferencias en los efectos adversos del Handihaler versus Respimat ${ }^{5}$. El mecanismo de acción de estos fármacos se debe a su unión prolongada a los receptores M1 y M3, por 14,6 y 34 h respectivamente, y su disociación rápida a las $4 \mathrm{~h}$ del receptor M2. Estudios clínicos en pacientes $\mathrm{EPOC}^{2,3}$ han demostrado que aporta una broncodilatación más sostenida, disminuye las exacerbaciones y hospitalizaciones, y mejora la disnea, la calidad de vida y la tolerancia al ejercicio (evidencia A) (Tabla 2).

Los efectos adversos más frecuentes de estos medicamentos son: sequedad de la boca (6-16\%), retención urinaria $(3 \%)$, náuseas $(3 \%)$, cefalea $(3 \%)$, estreñimiento $(10 \%)$. Deben usarse con precaución en pacientes con hiperplasia prostática, con arritmias, y aquellos con glaucoma de ángulo abierto.

Bromuro de Aclidinium. Aprobado el 24-72012 por la FDA de USA, actúa sobre los receptores muscarínicos, pero con una permanencia menor que el tiotropio, empleándose cada $12 \mathrm{~h}$ en dosis de 200 a $400 \mu \mathrm{g}$. Si bien, ha mejorado síntomas, $\mathrm{VEF}_{1}$ y calidad de vida, no hay todavía una suficiente experiencia clínica que lo avale completamente $^{6}$ y no está disponible en Chile.

Bromuro de Glicopirronio (Seebri $\left.{ }^{\circledR}\right)$. Se emplea con un dispositivo (Breezhaler), en una dosis 
diaria única de $50 \mu \mathrm{g}$, en EPOC moderada a severa. Tiene la ventaja sobre el tiotropio de un inicio de acción más rápido ( $5 \mathrm{~min}$ ), con un efecto máximo a las $2 \mathrm{~h}$. Produce una mejoría del $\mathrm{VEF}_{1}$ que es significativamente mayor que el placebo a las $12 \mathrm{~h}$, que se mantiene algo menor a las $24 \mathrm{~h}^{7}$. También muestra una mejoría significativa en el tiempo de tolerancia al ejercicio y disminución del índice de disnea matinal ${ }^{8}$. Finalmente en un estudio de 52 semanas versus tiotropio y placebo determinó un 34\% de reducción de las exacerbaciones en relación al placebo $(\mathrm{p}<0,003)^{9}$.

\section{Glucocorticoides inhalados}

Los corticoides inhalados tienen una gran actividad antiinflamatoria local, reduciendo la inflamación de la mucosa de las vías aéreas, con pocos efectos sistémicos debido a que se inactivan rápidamente en el hígado.

Toman algunas horas en actuar, debido a que deben penetrar primero al citoplasma celular para reaccionar con los receptores citosólicos, y luego penetrar al núcleo donde interfieren la transcripción, disminuyendo la síntesis de proteínas inflamatorias. Su efecto máximo se alcanza a los 3 a 5 días de su empleo, siendo el tratamiento controlador más efectivo en el asma (evidencia A), y es capaz de disminuir las exacerbaciones de las EPOC cuando se asocian a un $\beta_{2}$ agonista de acción prolongada ${ }^{10-12}$ (evidencia $\mathrm{A}$ ).

$\mathrm{Su}$ administración mantenida en el tiempo reduce el número de células inflamatorias activadas en las vías respiratorias, y con ello disminuye la hiperreactividad bronquial. El corticoide ideal sería aquel que tuviera una gran actividad local tópica con menos efectos sistémicos. Dependiendo de su farmacocinética (grado de inactivación en el tracto gastrointestinal, metabolización hepática) cada corticoide puede producir efectos secundarios sistémicos de distinta intensidad, Debido a su gran afinidad por la albúmina plasmática, es poco el corticoide libre disponible para producir reacciones adversas. Los factores que determinarán la biodisponibilidad pulmonar de los corticoesteroides inhalados son: la dosis diaria empleada, la frecuencia de su administración, la duración del tratamiento y el sistema de administración.

\section{Utilidad clínica de los corticoides inhalados (CEI) en el Asma estable}

Si bien se han empleado en varios tipos de afecciones pulmonares, sin duda donde han demostrado mayor utilidad es en el asma bronquial.
Los CEIs disponibles en el país son: beclometasona, budesonida, fluticasona, mometasona y ciclosenida, ya sea solos, o asociados a un $\beta_{2}$ agonista de acción prolongada, en dispositivos presurizados con dosis medida o como polvos secos (Tabla 3). Recientemente se ha introducido en el país el furoato de mometasona, bajo la forma de un polvo seco que se administra con un dispositivo twisthaler, que tiene un contador de dosis utilizadas y que se activa inmediatamente al retirar la tapa, lo que facilita su uso. Ya en los años 2003 y $2004^{13,14}$ se había valorado su eficacia y seguridad con el empleo de $400 \mu \mathrm{g} 1 \mathrm{vez}$ al día, en comparación con budesonida y fluticasona. Un metanálisis reciente ${ }^{15}$ ha corroborado su buen efecto controlador de síntomas y exacerbaciones, semejante a los regímenes terapéuticos que emplean budesonida o propionato de fluticasona.

La introducción reciente en el país de la asociación del furoato de mometasona a fumarato de formoterol, (Dulera $\left.{ }^{\circledR}\right)$ agrega una nueva arma terapéutica para controlar el asma persistente de leve a severo, con presentaciones de 100/5 y 200/5 $\mu \mathrm{g}$ por inhalación, 2 inhalaciones cada 12 $\mathrm{h}$ en pacientes mayores de 12 años.

En el futuro la combinación de furoato de fluticasona (100 ó $200 \mu \mathrm{g}$ con vilanterol $25 \mu \mathrm{g}$ ) administrados cada $24 \mathrm{~h}$ en pacientes de más de 12 años podría ser otra alternativa para el manejo del asma, dado su uso sin efectos adversos de significación ${ }^{16}$, pero deberá esperarse su aprobación por la FDA al respecto.

En el asma persistente los CEI constituyen el tratamiento más efectivo de mantención, tanto para lograr el control de la enfermedad, como para disminuir el riesgo de exacerbaciones agudas. En el asma persistente leve, basta una dosis baja, asociada a un $\beta_{2}$ agonista de acción corta para lograr el control de la afección (evidencia A). En el asma persistente moderado y severo, la dosis del corticoide inhalado será mediana o alta, asociada a $\beta_{2}$ agonista de acción prolongada (evidencia A). En general estas asociaciones son suficientes para lograr el control de la enfermedad, definida claramente en las Guías para el manejo del asma de GINA de $2011^{17}$.

Solamente en el asma severo, resistente a dosis altas del corticoesteroide inhalado asociado a un $\beta_{2}$ agonista de larga acción (alrededor de un 5\%), deben considerarse otros medicamentos controladores, como los inhibidores de leucotrienos y teofilina de acción sostenida, o agregar un corticoide oral si tampoco se logra el control de la afección con los anteriores (evidencia B).

Últimamente, se ha demostrado la eficacia de la asociación de tiotropio a los corticoides inha- 
lados y un beta agonista de acción prolongada, para controlar el asma severo resistente a corticoides, ya sea con $18 \mu \mathrm{g}$ del Handihaler ${ }^{18}$ o $5 \mu \mathrm{g}$ de Respimat ${ }^{19}$.

Las pautas de administración de corticoides inhalados se presentan en la Tabla 3.

\section{Tratamiento inhalatorio de la EPOC estable}

Dado la heterogeneidad y complejidad de la presentación clínica de la EPOC estable, especialmente los síntomas, su grado de obstrucción de las vías aéreas medidas por $\mathrm{VEF}_{1} / \mathrm{CVF}$, y la frecuencia y severidad de las exacerbaciones, inicialmente fue muy difícil precisar cuál sería el tratamiento más conveniente para cada una de estas eventualidades. El primer acercamiento fue el Consenso Gold de $2007^{20}$, que en base a las cifras del $\mathrm{VEF}_{1} / \mathrm{CVF}$ después del broncodilatador clasificó a las EPOC en grados I (leve), II (moderada); III (severa) y IV (muy severa), de acuerdo si el $\mathrm{VEF}_{1}$ era $\geq 80 \%$; $\leq$ el 70 y $>50 \%$; $\leq 50$ y $>30 \%$ y $<30 \%$ respectivamente.

Si bien esta clasificación era muy útil dado su simplicidad, adolecía de severas limitaciones: 1) $\mathrm{VEF}_{1} / \mathrm{CVF}$ no guarda una relación adecuada con la cuantía de los síntomas; 2) tampoco es útil su correlación con la calidad de vida ${ }^{21}$ y 3 ) la heterogeneidad de cada variable tampoco tiene una relación con los síntomas (por ejemplo la disnea), y aún más, las exacerbaciones y calidad de vida se relacionan de distinta forma en cada paciente, como se demostró en el estudio Eclipse ${ }^{22}$. Por todos estos inconvenientes, la presentación de una clasificación "combinada", que incluye la severidad de los síntomas, la frecuencia y gravedad de las exacerbaciones, además de la obstrucción de las vías aéreas $\left(\mathrm{VEF}_{1} / \mathrm{CVF}\right)$, se presentó en los Consensos Gold de 2011 y $2013^{23}$. El fin de esta nueva visión de las EPOC fue crear distintos tratamientos de acuerdo con la complejidad de la EPOC y de acuerdo con ello reducir la sintomatología, aumentar la tolerancia al ejercicio, mejorar el estado de salud y calidad de vida, y reducir el riesgo de progresión de la enfermedad y muerte, mediante la prevención y control de las exacerbaciones. Esta nueva clasificación combinada de la EPOC toma en cuenta la evaluación de los síntomas, el grado de limitación del flujo aéreo (medido por espirometría) y el riesgo de exacerbaciones fijando un puntaje a cada una de estas tres variables, para llegar a una categorización de la EPOC como A-B-C-D, a las cuales se le aplicarán medidas de tratamiento de creciente complejidad (Figura 1).

De acuerdo con los puntajes de la limitación del flujo aéreo (1 a 4), frecuencia de exacerbaciones (0-2), más la evaluación de los síntomas (mMRC y CAT) los pacientes se clasifican en 4 grupos, los A con pocos síntomas y escasa obstrucción de las vías aéreas y sin exacerbaciones; el B con mayores síntomas y obstrucción de las vías aéreas y pocas exacerbaciones, el $\mathrm{C}$ con pocos síntomas, pero con mayor obstrucción de las vías aéreas y exacerbaciones frecuentes y el D con más severidad de síntomas, mayor obstrucción de las vías aéreas y aumento de la frecuencia de exacerbaciones.

Las EPOC A con pocos síntomas (disnea mMRC 0-1 y CAT $<$ de 10 ), clasificación espirométrica $0-1$ y sin exacerbaciones, se beneficiarán con los broncodilatadores de corta acción ( $\beta_{2}$ agonista de acción corta o ipratropio prn o a dosis fijas 3 a 4 veces al día. La EPOC $\mathrm{B}$ de bajo riesgo, pero con mayores síntomas (mMRC mayor o igual a 2 y CAT mayor o igual a 10), con una evaluación espirométrica GOLD 1-2 (60 a 50\%) se beneficiarán con el uso de un $\beta_{2}$ agonista de larga acción (LABA) o un anticolinérgico de larga acción (LAMA). El grupo $\mathrm{C}$, de mayor riesgo pero con pocos síntomas, y con obstrucción GOLD 3-4 (30-50\%) y dos o más exacerbaciones, responderían mejor a las asociaciones de LABA + LAMA o de un corticoide inhalado + un LABA.

El grupo D de alto riesgo, con más síntomas y obstrucción de vías aéreas (GOLD menor del $30 \%$ ) y 2 ó más exacerbaciones y hospitalizaciones anuales, requerirán de un tratamiento triasociado: $\mathrm{LABA}+\mathrm{LAMA}+\mathrm{CEI}^{23}$. Una alternativa recientemente publicada ${ }^{24}$ pero que todavía no ha sido juzgada por los meta-análisis, es la asociación de glicopirronio $(50 \mu \mathrm{g})+$ indacaterol $(150-300 \mu \mathrm{g})$ en la EPOC moderada a severa. Según Wedzicha y cols, los resultados del estudio $\mathrm{SPARK}^{24}$ (randomizado y doble ciego), que evaluó a tres grupos de tratamiento: glicopirronio + indacaterol, (QVA 149); glicopirronio solo y tiotropio solo, cuyo objetivo primario era evaluar si QVA 149 era mejor que las otras dos alternativas de tratamiento, mostró un resultado superior del QVA 149 al empleo de monoterapia con glicopirronio y tiotropio. En síntesis, la asociación glicopirronio + indacaterol superó en un más $12 \%$ al glicopirronio en la reducción de las exacerbaciones, y tuvo una mejoría significativa del $\mathrm{VEF}_{1}$ (70-80 ml; $\mathrm{p}<0,001)$ en relación a glicopirronio y tiotropio por separado.

Debemos dejar constancia que estas orientaciones terapéuticas no consideran nuevas asociaciones en vías de aprobación por la FDA y ya aprobadas en Europa. 


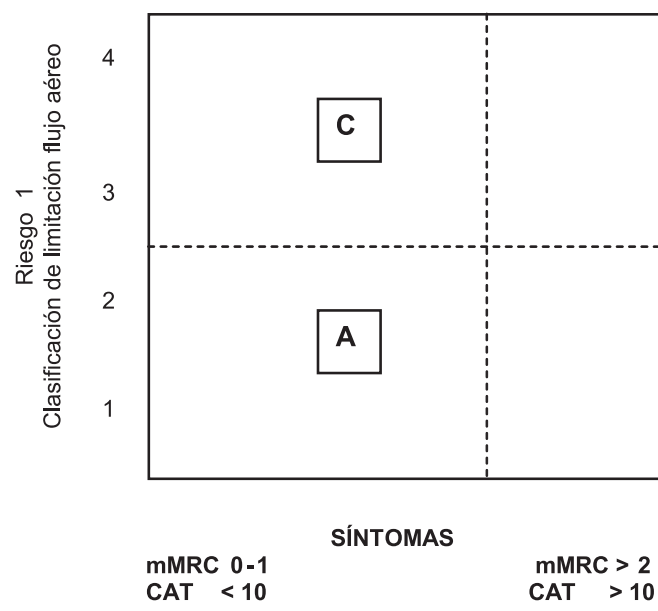

2

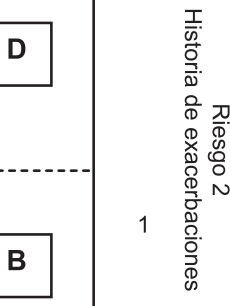

0

Categoración de la EPOC

\begin{tabular}{|c|l|c|c|c|c|}
\hline $\begin{array}{c}\text { Categoría } \\
\text { del paciente }\end{array}$ & Características & $\begin{array}{c}\text { Clasificación } \\
\text { espirométrica }\end{array}$ & $\begin{array}{c}\text { Exacerbación } \\
\text { por año }\end{array}$ & mMRC $^{*}$ & CAT $^{\#}$ \\
\hline A & $\begin{array}{l}\text { Riesgo bajo: } \\
\text { menos síntomas }\end{array}$ & GOLD 1-2 & $\leq 1$ & $0-1$ & $<10$ \\
\hline B & $\begin{array}{l}\text { Riesgo bajo: } \\
\text { más síntomas }\end{array}$ & GOLD 1-2 & $\leq 1$ & $\geq 2$ & $\geq 10$ \\
\hline C & $\begin{array}{l}\text { Riesgo alto: } \\
\text { menos síntomas }\end{array}$ & GOLD 3-4 & $\geq 2$ & $0-1$ & $<10$ \\
\hline D & $\begin{array}{l}\text { Riesgo alto: } \\
\text { más síntomas }\end{array}$ & GOLD 3-4 & $\geq 2$ & $\geq 2$ & $\geq 10$ \\
\hline
\end{tabular}

*Modified Medical Research Council dyspnea scale.

\#COPD Assessment test score (calidad de vida).
Figura 1. Evaluación combinada de la EPOC. Asociación entre síntomas, clasificación espirométrica y futuros riesgos de exacerbación (GOLD 2013) ${ }^{23}$.
Se trata de las combinaciones de Respimat tiotropio $(2,5 \mu \mathrm{g})$ con Olodaterol $(5 \mu \mathrm{g})$ : (LAMA + LABA), que se emplea como una inhalación cada 24 h con un buen efecto terapéutico ${ }^{25}$, y la asociación del Furoato de Fluticasona $(100 \mu \mathrm{g})+$ Vilanterol $(25 \mu \mathrm{g})\left(\right.$ CEI de larga acción + un $\beta_{2}$ agonista de acción prolongada, ambos de $24 \mathrm{~h}$ ), y cuya eficacia y seguridad han sido demostradas en varios estudios ${ }^{26,27}$. En una revisión recien$\mathrm{te}^{28}$ se comunica que estas asociaciones fueron aprobadas para su uso en EPOC en USA. Otra asociación farmacológica importante de conocer, y en etapa final de evaluación de la FDA, es la combinación del Umeclidinio (LAMA) con el Vilanterol (LABA) ambos de $24 \mathrm{~h}$ de duración, formulado en dosis de $62,5 / 25 \mu \mathrm{g}$ y $125 / 25 \mu \mathrm{g}$, una vez al día para pacientes con EPOC. Su eficacia y seguridad ha sido evaluada por dos estudios ${ }^{29}$ multicéntricos de 24 y 52 semanas, versus tiotropio handihaler $(18 \mu \mathrm{g})$ en 842 pacientes EPOC moderados a severos. En el estudio de 24 semanas el $\mathrm{VEF}_{1}$ valle mostró una mejoría de $74 \mathrm{ml}$ superior a la observada con tiotropio $(\mathrm{p}<0,003)$, en los pacientes que recibieron $125 / 25 \mu \mathrm{g}$ de umeclidinio/vilanterol.

Finalmente, debemos mencionar la experiencia con GSK 961081 un medicamento excepcional, que en una sola molécula presenta un antagonismo muscarínico y una actividad broncodilatadora $\beta$-2 agonista (MABA). En un estudio multicéntrico, randomizado y doble ciego versus placebo o salmeterol, de 4 semanas, que comprendió 436 pacientes con EPOC moderado a severo, que recibieron 2 inhalaciones de $200 \mu \mathrm{g}$ o una de $400 \mu \mathrm{g}$, una vez al día, tuvieron una mejoría del $\mathrm{VEF}_{1}$ valle de 215 y $249 \mathrm{ml}$ respectivamente, que fueron estadísticamente significativas en relación a los controles ${ }^{30}$. No hubo efectos secundarios cardiovasculares de importancia con esta nueva terapia bifuncional.

\section{Orientación práctica en el tratamiento de la EPOC estable}

- Tomar en cuenta que el tratamiento inhalado es de elección en esta etapa de las EPOC.

- La elección de un $\beta$ agonista, anticolinérgico 
o un corticoide inhalado solo o en asociación, depende de la disponibilidad de los medicamentos, del grado de severidad de la afección y la respuesta individual de cada paciente en cuanto al alivio de los síntomas y probables efectos adversos. A este respecto, deben tomarse en cuenta las co-morbilidades asociadas, si el enfermo es hipertenso, adolece de arritmias, ha tenido afecciones coronarias, es diabético, tiene antecedentes de tuberculosis pulmonar o presenta cicatrices residuales pulmonares.

- Para mejorar los síntomas es más conveniente y efectivo el tratamiento permanente con broncodilatadores de acción prolongada (LABA o LAMA), que además reducen las exacerbaciones, hospitalizaciones y mejoran la calidad de vida, por lo cual deberían estar siempre presentes en cualquier esquema ${ }^{28}$.

- La combinación de un LABA con un LAMA puede mejorar la eficacia y reducir el riesgo de efectos adversos, en comparación con aumentar la dosis del broncodilatador beta 2 agonista $^{28}$.

- Debe tenerse cuidado cuando se emplean combinaciones de mayor riesgo, como LABA de $24 \mathrm{~h}$ con medicamentos que asocian un CEI + LABA, por la probabilidad de accidentes cardiovasculares. Igualmente el empleo de CEI solos o asociados a LABA, por la mayor frecuencia de neumonías $(60 \%)$ y de tuberculosis si existe este antecedente ${ }^{28}$.

- El tratamiento con Tiotropio u otro LAMA (glicopirronio o umeclidinio) + un LABA de $24 \mathrm{~h}+$ un corticoide inhalado de larga acción (furoato de fluticasona), confiere beneficios adicionales al esquema en las EPOC más severas.

- El empleo de un LAMA de mayor potencia (Glicopirronio) + un LABA de $24 \mathrm{~h}$ (Indacaterol), unido o no a un CEI de acción prolongada, podría mejorar el futuro de las EPOC muy severas ${ }^{26}$.

Debido a la tendencia a personalizar lo más posible el tratamiento del paciente de acuerdo con la característica de la enfermedad, se ha creado una clasificación fenotípica de la EPOC, a semejanza de lo que se ha hecho en el asma bronquial. El fenotipo puede ser un atributo simple o una combinación de atributos de la enfermedad que describa una diferencia entre individuos con EPOC. Pueden relacionarse con características clínicas significativas, como los síntomas, exacerbaciones, respuesta a la terapia, velocidad de la progresión, tendencia a la exacerbación o ser cuadros mixtos de asma y EPOC.
La Sociedad Española de Patología Respiratoria (SEPAR) ha creado una Guía para el manejo de la EPOC, en que ha aplicado las características fenotípicas más importantes de la EPOC para establecer una nueva clasificación y orientación terapéutica ${ }^{31}$.

La diferencia con la clasificación GOLD 2013 es que establece el término agudizador en vez de exacerbación y propone 5 fenotipos (Figura 2): el no agudizador (A), el agudizador enfisematoso (C), el agudizador bronquítico crónico (D) y el mixto EPOC-ASMA (B), que se cataloga así por su alta reversibilidad funcional con el broncodilatador y eosinofilia en el esputo. Esta Guía se estructura según sus fenotipos clínicos y se modula en 4 niveles de I a IV, de acuerdo con la gravedad del cuadro (Tabla 4 y Figura 2).

\section{Fenotipos clínicos de la EPOC y su tratamiento según la guía española}

Para el manejo de EPOC propone diferentes tratamientos de acuerdo con el fenotipo, destacándose que sólo en el grupo A, enfisema o bronquítico sin agudizaciones (inflamación), sugiere el empleo de solo broncodilatadores, LABA (salmeterol o indacaterol) primero, y si no hay respuesta anticolinérgicos (tiotropio $u$ otro LAMA). En el fenotipo Asma-EPOC, grupo B, agrega a los anteriores un antinflamatorio inhalado (tratamiento doble: LABA + CEI, o LABA + LAMA), y establece uno triple (LABA + LAMA + CEI) cuando el fenotipo es enfisema o bronquítico agudizadores con evidente inflamación (grupos C y D) (Tabla 4).

Estos cambios de estrategia, abordando el tratamiento según fenotipos, tendería a llegar al ideal de un tratamiento personalizado para cada paciente, con una base de broncodilatadores de acción prolongada a los cuales se agregarían otros fármacos según cuál sea el fenotipo y su severidad. Además establece prestar atención a las comorbilidades optimizando su control. El objetivo de estos tratamientos personalizados es reducir los síntomas crónicos de la enfermedad y prevenir las exacerbaciones (objetivo a corto plazo), y aumentar la sobrevida (objetivo a largo plazo) al reducir los riesgos.

Al terminar esta revisión, creemos haber aclarado el renovado panorama del tratamiento inhalatorio del asma y EPOC, sobre todo de esta última, una entidad olvidada terapéuticamente por mucho tiempo, en la cual disponemos actualmente de un amplio arsenal farmacológico, que es necesario conocer para aplicar un tratamiento personalizado óptimo. 
Tabla 4. Guía española para el manejo de la EPOC estable moderada a severa.

Tratamiento farmacológico de la EPOC según fenotipos y niveles I a IV de gravedad*

\begin{tabular}{|c|c|c|c|c|}
\hline \multirow[b]{2}{*}{ Fenotipo } & \multicolumn{4}{|c|}{ Nivel de gravedad } \\
\hline & I & II & III & IV \\
\hline $\begin{array}{c}\text { A } \\
\text { No agudizador con } \\
\text { enfisema o con BC }\end{array}$ & $\begin{array}{l}\text { LAMA o LABA } \\
\text { SABA o SAMA }\end{array}$ & $\begin{array}{l}\text { LAMA o LABA } \\
\text { LAMA + LABA }\end{array}$ & LAMA + LABA & $\begin{array}{c}\text { LAMA + LABA + } \\
\text { Teofilina }\end{array}$ \\
\hline $\begin{array}{c}\text { B } \\
\text { Mixto EPOC-Asma }\end{array}$ & $\mathrm{LABA}+\mathrm{CEI}$ & $\mathrm{LABA}+\mathrm{CEI}$ & $\begin{array}{c}\mathrm{LAMA}+\mathrm{LABA}+ \\
\mathrm{CEI}\end{array}$ & $\begin{array}{c}\text { LAMA + LABA + } \\
\text { CEI + Teofilina }\end{array}$ \\
\hline $\begin{array}{c}\text { C } \\
\text { Agudizador con } \\
\text { enfisema }\end{array}$ & LAMA o LABA & $\begin{array}{c}\text { (LABA o LAMA) } \\
+ \text { CEI }\end{array}$ & $\begin{array}{c}\mathrm{LAMA}+\mathrm{LABA}+ \\
\mathrm{CEI}\end{array}$ & $\begin{array}{c}\mathrm{LAMA}+\mathrm{LABA}+ \\
\mathrm{CEI}\end{array}$ \\
\hline$\frac{\text { D }}{\text { Agudizador con BC }}$ & LAMA o LABA & $\begin{array}{c}\text { (LAMA o LABA) } \\
+ \text { (CEI o IPE4) } \\
\text { LAMA + LABA } \\
\text { LAMA o LABA }\end{array}$ & $\begin{array}{c}\text { LAMA + LABA + } \\
(\text { CEI o IPE4) } \\
(\text { LAMA o LABA) + } \\
\text { CEI + IPE4 }\end{array}$ & $\begin{array}{c}\mathrm{LAMA}+\mathrm{LABA}+ \\
\text { CEI } \\
+ \text { IPE4 }\end{array}$ \\
\hline
\end{tabular}

*Adaptado de Miravitlles et $\mathrm{al}^{30}$. BC: bronquitis crónica; SABA: $\beta-2$ agonista de corta duración; SAMA anticolinérgico de corta duración; CEI: corticosteroide inhalado; LAMA: anticolinérgico de larga duración; LABA: $\beta-2$ agonista de larga duración; IPE4: inhibidor de la fosfodiesterasa.

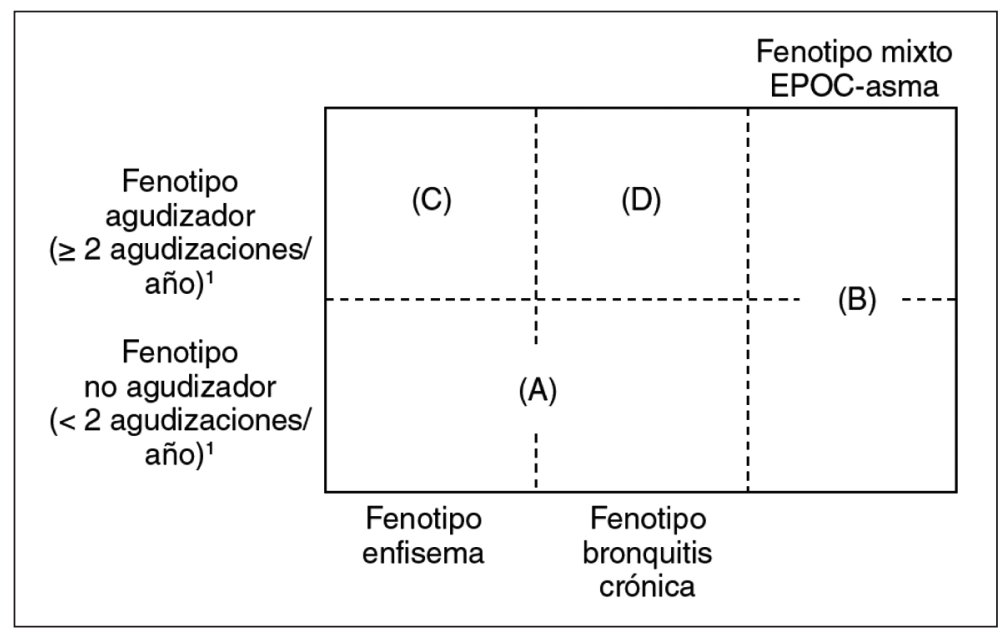

Figura 2. Fenotipos clínicos de EPOC según Ges EPOC ${ }^{31}$ (ver texto).

\section{Bibliografia}

1.- KERWIN E M, MELI J, PECKIT C, LASSEN C, KRAMER B, FILCEK S. Efficacy and safety of Indacaterol $75 \mu \mathrm{g}$ once daily in patients with moderate to severe COPD Am J Crit Care Med 2011; 14: A 1595.

2.- NIEWOEHNER D E, RICE K, COTE C, PAULSON D, COOPER JA, KORDUCKI L, et al. Prevention of exacerbations of chronic obstructive pulmonary disease with tiotropium, a once daily inhaled anticholinergic bronchodilator: a randomized trial Ann Inter Med 2005; 143: 317-26.
3.- TASHKIN D P, CELLI B, SENN S, BURKHART D, KESTEN S, MENJOBE S, et al. A 4 year trial of tiotropium in chronic obstructive pulmonary disease N. Engl J Med 2008; 359: 1543-54.

4.- SINGH S, LOKE Y K, ENRIGHT P L, FURBERG C D. Mortality associated with tiotropium mist inhaler in patients with chronic obstructive pulmonary disease: sistematic rewiew and meta-analysis of randomized control trials. BMJ 2011; 342: d3215. doi 10. 1/36/bmj. d3215. Review

5.- WISE R A, ANZUETO A, COTTON D, DAHL R, DEVINS T, DISSE B, et al. Tiotropium Respimat Inhaler 
and the risk of death in COPD N. Engl J Med 2013; 369: 1491-501.

6.- FUHR R, MAGNUSSEN H, SAREM C, RIBERA A, KINSTEN A M, FALQUE M, et al. Efficacy of Aclidinium bromide $400 \mu \mathrm{g}$ twice daily compared with placebo and tiotropium in patients with moderate to severe COPD. Chest 2012; 141: 745-52.

7.- D'URZO A, FERGUSON G T, VAN NOORD J A, HIRATA K, MARTIN C, HORTON R, et al. Efficacy and safety of once daily NVA 237 in patients with moderate to severe COPD: the GLOW 1 trial. Resp Res 2011; 12 : 156. doi: 10.1186/1465-9921-12-156

8.- BEEH K M, SINGH D, DI SCALA L, DROLLMANN A. Once daily NVA 237 improves exercise tolerance from the first dose in patients with COPD: the GLOW 3 trial. International J. COPD 2012; 7 : 503-13.

9.- KERWIN E, HEBERT J, GALLAGHER N, MARTIN C, OVEREND T, ALAGAPPAN V K, et al. Efficacy and safety of NVA 237 versus placebo and tiotropium in patients with COPD: the GLOW 2 study. Eur Respir J 2012; 40: 1100-14.

10.- VESTBO J. The TORCH (towards a revolution in COPD health) survival study protocol. Eur Respir J 2004; 24: 206-10.

11.- CALVERLY P M, ANDERSON JA, CELLI B, FERGUSON G T, JENKINS C, JONES P W, et al. Salmeterol and Fluticasone propionate and Survival in Chronic Obstructive Pulmonary Disease. N Eng J Med 2007; 356: 775-89.

12.- RABE KF. Treating COPD-The Torch trial, P Values and the Dodo. Editorial N. Engl J Med 2007; 356: 8514.

13.- CORREN J, BERKOWITZ R, MURRAY J J, PRENNER B. Comparison of mometasone furoate once a day versus budesonide once a day in patients with moderate persistent asthma. Int J Clin Pract 2003; 57 : 567-72.

14.- WARDLAW A, LARIVEE P, ELLER J, CROCKROFT DW, GHALY L, HARRIS A G. Efficacy and safety of mometasone furoate dry powder inhaler $v s$ fluticasone propionate metered-dose inhaler in asthma subjects previously using fluticasone propionate. Ann Allergy Asthma Immunol 2004; 93: 49-55.

15.- YANG D, WANG J, BUNJHOO H, XIONG W, XU Y, ZHAO J. Comparison of the efficacy and safety of mometasone furoate to other inhaled steroids for asthma: a metanalysis. Assian Pacif J. Allergy Immunol 2012; 31: 26-33.

16.- BUSSE W, O 'BYRNE P, BLEECKER E, LÖTWALL J, WOODCOCK A, ANDERSEN L, et al. Safety and tolerability of the novel inhaled corticosteroid fluticasone furoate in combination with the Beta 2 agonist vilanterol administered once daily for 52 weeks in patients $>$ of 12 years old with asthma: a randomized trial. Thorax 2012; 68: 513-20.
17.- Evidence based Strategy for Asthma management and prevention. Global Initiative for Asthma revised 2011 www.ginasthma.org.

18.- PETERS S, KUNSELMAN S, ICITOVIC N, MOORE W, PASCUAL R, AMENENDES B, et al. Tiotropium bromide step-up therapy for adults with uncontrolled asthma. N. Engl J Med 2010; 363: 1715-26.

19.- KERSTJENS H, ENGEL M, DAHL R, PAGGIARO P, BECK E, et al. Tiotropium in asthma poorly controlled with standard combination therapy. N. Engl J. Med 2012; 367: 1198-200.

20.- RABE K F, HURD S, ANZUETO A, BARNES P J, BUIST S A, CALVERLEY P, et al. Global strategy for the diagnosis, management and prevention of chronic obstructive pulmonary disease: GOLD executive summary. Am J Respir Crit Care Med 2007; 176: 53255.

21.- JONES PW. Health status measurement in chronic obstructive pulmonary disease. Thorax 2001; 56: 8807.

22.- VESTBO J, ANDERSON W, COXSON H O, CRIM C, DAWBER F, EDWARDS L, et al. Evaluation of COPD longitudinally to identify predictive surrogate end points (ECLIPSE) Eur Resp J 2008; 31: 869-73.

23.- VESTBO J, HURD S S, AGUSTÍ AG, JONES PW, VOGELMEIER C, ANZUETO A, et al. Strategy for the diagnosis, management and prevention of chronic obstructive pulmonary disease GOLD executive summary Am J Crit Care Med 2013; 187: 347-65.

24.- WEDZICHA JA, DECRAMER M, FISKER J H, NIEWOEHNER DE, SANDSTRÖM T, TAYLOR $\mathrm{AF}$, et al. Analysis of chronic obstructive pulmonary disease exacerbations with the dual bronchodilator QVA 149 compared with glycopirronium and tiotropium (Spark), a randomized double blind parallel group study Lancet Respiratory Medicine 2013; 1: 199-209.

25.- AALBERS R, MABEKI-YASDI M R, HAMILTON A, WALTETE-WIJKER S, PIROVAROVA A, SCHMIDT $\mathrm{O}$, et al. Dose finding study for tiotropium and olodaterol when administered in combination via the Respimat inhaler in patients with COPD Eur Respir Journal 2012; 40 (suppl 56) abstract 62882.

26.- DRANSFIELD M T, BOURBEAU J, JONES P W, HANANIA N A, MAHLER D A, VESTBO J, et al. One day inhaled fluticasone furoate and vilanterol versus vilanterol only for prevention of exacerbations of COPD: two replicate double blind parallel group randomized controlled trials Lancet Respiratory Medicine 2013; 1 : 210-23.

27.- MARTÍNEZ F J, BOSCIA J, FELDMAN G, SCOTTWILSON C, KILBRIDE S, FABBRI L, et al. Fluticasone furoate/vilanterol $(100 / 25 ; 200 / 25 \mu \mathrm{g})$ improves lung function in COPD, a randomized trial Resp Med 2013; 107: 550-9.

28.- TASHKIN D P, FERGUSON G T. Review combination 
bronchodilator therapy in the management of chronic obstructive pulmonary disease Respir Res 2013; 14: 49-66.

29.- FELDMAN GF, EDIN A. The combination of Umeclidinium bromide and vilanterol in the management of Chronic Obstructive Pulmonary Disease: current evidence and future prospects. Ther Adv Respir Dis 2013; 7: 311-9.

30.- WIELDERS P, LUDWIG-SERGPIEL A, LOCAN-
TORE N, BAGGEN S, CHAN R, RILEY J H. A new class of bronchodilator improves lung function in COPD, a trial with GSK 961081. Eur Respir J 2013; 42: 972-81.

31.- MIRAVITLLES M, SOLER-CATALUÑA JJ, CALLE M, MOLINA J, ALMAGRO P, QUINTANO JA, et al. Guía Española de la EPOC (Ges EPOC) Tratamiento farmacológico de la EPOC estable. Arch Bronconeumol 2012; 48: 247-57.

Correspondencia a:

Dr. Edgardo Carrasco C.

Email: edgardo@bronco-pulmonar.cl 